\title{
IDEMPOTENT MEASURES ON A COMPACT TOPOLOGJCAL SEMIGROUP
}

\author{
M. HEBLE AND M. ROSENBLATT ${ }^{1}$
}

1. Introduction. Let $S$ be a compact topological (Hausdorff) semigroup. Consider any probability measure $\nu$ regular on $S$. Some of the limit properties of the average of the convolution sequence

$$
\frac{1}{n} \sum_{j=1}^{n} \nu^{(j)}=\nu_{n}
$$

were discussed in [5]. Let $\Sigma(\nu)$ be the support (or spectrum) of $\nu$. One can just as well take $S$ as the closure of $U_{n}(\Sigma(\nu))^{n}=S(\nu)$ since all the convolutions $\nu^{(j)}$ are concentrated on $S(\nu)$. It was shown that $\lim _{n \rightarrow \infty} \nu_{n}=\mu$ exists in the sense that $\lim _{n \rightarrow \infty} \int f d \nu_{n}=\int f d \mu$ for every continuous $f$ on $S$. Further, $\mu$ is regular with support the kernel $K$ (minimal two-sided ideal) of $S(\nu)$ and is idempotent, that is,

$$
\mu^{(2)}=\mu .
$$

In [5] the definition of the convolution of two regular measures $\nu, \mu$ on $S$ was introduced as follows. Let $B=B(S)$ be the Borel field generated by the open sets of $S$. If $B \in B(S), \nu * \mu(B)$ was given by

$$
\nu * \mu(B)=\int c_{B}(\nu u)(\nu \times \mu)(d(v, u))
$$

where $c_{B}$ is the characteristic function of the set $B$ and $\nu \times \mu$ is the product measure generated by $\nu, \mu$ on $S \times S$. This is not valid for all compact Hausdorff semigroups since $A_{B}=\{(v, u) \mid v u \in B\}$ may not be in the product Borel field $B(S) \times B(S)$ even though $B \in B(S)$. It is valid for separable Hausdorff semigroups. However, one can generally introduce the convolution of two regular measures $\nu, \mu$ on a compact Hausdorff semigroup $S$ as follows. Given any continuous $f$ on $S$, let

$$
\int\left\{\int f(v u) \nu(d v)\right\} \mu(d u)=L(f) .
$$

This defines a continuous functional of the continuous functions on $S$. By the Riesz theorem [1] on such functionals, there is a regular

Presented to the Society July 12, 1961; received by the editors July 14, 1961 and, in revised form, December 27, 1961.

1 This research was supported by the Office of Naval Research. Reproduction in whole or in part is permitted for any purpose of the United States Government. 
measure on $B(S)$ determined by $L(f)$

$$
L(f)=\int f(u)(\nu * \mu)(d u)
$$

which we shall call the convolution $\nu * \mu$ of $\nu, \mu$. With this definition of the convolution $\nu * \mu$, the proofs of the results cited in [5] can be simply modified so as to hold for the case of a general compact Hausdorff semigroup $S$. Kernel semigroups $K$ are rather special semigroups and are often referred to as completely simple semigroups [6]. Every compact completely simple semigroup can be represented as the product space $T \times X \times Y$ of a compact topological group $T$ and compact Hausdorff spaces $X, Y$ where the multiplication of two elements $s=(t, x, y), s^{\prime}=\left(t^{\prime}, x^{\prime}, y^{\prime}\right)$ is given by

$$
s s^{\prime}=(t, x, y)\left(t^{\prime}, x^{\prime}, y^{\prime}\right)=\left(t \phi\left(x, y^{\prime}\right) t^{\prime}, x^{\prime}, y\right)
$$

with $\phi$ a continuous function on the product space $X \times Y$ into $T$ [6]. We can therefore identify $K$ with such a space $T \times X \times Y$ and the corresponding $\phi$ function.

In [5] it was shown that every idempotent measure $\mu$ on a finite completely simple semigroup $K$ with support the whole semigroup is a $\bar{\mu}$ measure, that is, $\mu$ is a product measure

$$
\bar{\mu}=\chi \times \alpha \times \beta
$$

where $\chi$ is the normed Haar measure $(\chi(T)=1)$ of the finite group $T$ and $\alpha$ and $\beta$ are probability measures on $X$ and $Y$ respectively. This note extends the above result to any compact Hausdorff semigroup.

TheOREM. Let $\mu$ be a regular idempotent probability measure on a compact Hausdorff semigroup. Then $\mu$ has a completely simple subsemigroup $K$ as its support. Further $\mu$ is a $\bar{\mu}$ measure so that if $K$ has the representation $T \times X \times Y$ then on $B(T) \times B(X) \times B(Y)$

$$
\mu=\chi \times \alpha \times \beta
$$

where $\chi$ is the normed Haar measure of the group $T$ and $\alpha, \beta$ are regular probability measures on $X, Y$ respectively.

COROLlaRy. Given a regular measure $\nu$ on the compact Hausdorff semigroup $S$, the sequence of averaged convolution measures

$$
\nu_{n}=\frac{1}{n} \sum_{j=1}^{n} \nu^{(j)}
$$

converges to a $\bar{\mu}$ measure with support the kernel of $S(\nu)$. 
2. Proof of the theorem. We shall make use of a number of lemmas to prove the theorem. For convenience let

$$
A s^{-1}=\left\{s^{\prime} \mid s^{\prime} s \in A\right\} \text {. }
$$

LEMMA 1. Let $\mu$ be a regular probability measure on the compact Hausdorf semigroup $S$. Then for each $s \in S$, the measure $\mu\left(A s^{-1}\right)$, $A \in \mathbb{B}(S)$, is regular on $S$.

Here $B(S)$ is the Borel field generated by the open sets on $S$. By the regularity of $\mu$ on $S$, given any $\epsilon>0$, there is a closed set $C \subset A s^{-1}$ such that $\mu\left(A s^{-1}-C\right)<\epsilon$. Let $B=C s$. By the continuity of the multiplicative operation, we see that $B$ is closed. Further $B \subset A$. Thus $\mu\left((A-B) s^{-1}\right)<\epsilon$ since $C \subset B s^{-1} \subset A s^{-1}$.

LEMma 2. If $A \in B(S)$ and $\mu$ is a regular measure on the compact Hausdorff semigroup $S, \mu\left(A s^{-1}\right)$ is a Borel measurable function of $s$.

Let $\eta$ denote the class of continuous functions $f$ on $S$ with $0 \leqq f \leqq 1$. Further, given any set $A$, let $\bar{A}$ denote the complement of $A$. First let $O$ be an open set. Set

$$
A_{\alpha}=\left\{s \mid \mu\left(O s^{-1}\right)>\alpha\right\} \text {. }
$$

Now by the regularity of $\mu\left(\cdot s^{-1}\right)$ (see $[1 ; 2]$ )

$$
\mu\left(O s^{-1}\right)=\sup _{\delta \in \eta ; f=0 \text { on } \delta} \int f(u) \mu\left(d u s^{-1}\right)=\sup _{\delta \in \eta ; f=0 \text { on } \delta} \int f(u s) \mu(d u) .
$$

Given any $s \in A_{\alpha}$ there is an $\epsilon>0$ such that $\mu\left(O s^{-1}\right)>\alpha+\epsilon$. But then there is a function $f_{s} \in \eta$ with $f_{s}=0$ on $\bar{O}$ such that

$$
\int f_{s}(u s) \mu(d u)>\alpha+\epsilon / 2 \text {. }
$$

The set of points $\left\{z \mid \int f_{s}(u z) \mu(d u)>\alpha+\epsilon / 2\right\}$ is an open set containing $s$ and is a subset of $A_{\alpha}$. Hence $A_{\alpha}$ is open. This implies that $\mu\left(O s^{-1}\right)$ is Borel measurable in $s$.

The open sets are a field. Further the class of sets $A \in \mathbb{B}(S)$ for which $\mu\left(A s^{-1}\right)$ is Borel measurable is a monotone class. Hence this class is a Borel field and must coincide with $B(S)$ (see [4]).

Lemma 3. Given any set $A \in B(S)$ and $\nu$, $\mu$ regular probability measures on the compact Hausdorff semigroup $S$,

$$
\nu * \mu(A)=\int \nu\left(A s^{-1}\right) \mu(d s)
$$


It is enough to show this for an open set $O$ since it will then follow for general $A \in \mathbb{B}(S)$ by the regularity of $\nu * \mu$. Now

$$
\begin{aligned}
\nu * \mu(O) & =\sup _{\delta \in \text {; } f=0 \text { on } \delta} \int\left\{\int f(v u) \nu(d v)\right\} \mu(d u) \\
& \leqq \int\left\{\sup _{j \in \eta ; f=0 \text { on } \delta} \int f(v u) \nu(d v)\right\} \mu(d u)=\int \nu\left(O s^{-1}\right) \mu(d s) .
\end{aligned}
$$

Consider any fixed $\epsilon>0$. Let $A_{k, n}=\left\{s \mid k / 2^{n} \leqq \nu\left(O s^{-1}\right)<(k+1) / 2^{n}\right\}$, $k=0,1, \cdots, 2^{n}$, with $2^{-n+2}<\epsilon$. Then

$$
\left|\sum_{k=0}^{2^{n}} \frac{k}{2^{n}} \mu\left(A_{k, n}\right)-\int \nu\left(O s^{-1}\right) \mu(d s)\right|<\frac{1}{2^{n}} .
$$

There is a closed set $C_{k, n} \subset A_{k, n}$ with

$$
\mu\left(A_{k, n}-C_{k, n}\right)<\epsilon / 2^{n+2}, \quad k=0,1, \cdots, 2^{n} .
$$

Given any $s \in C_{k, n}$, there is a continuous function $f_{s} \in \eta, f_{s}=0$ on $\bar{O}$, such that

$$
\int f_{v}(v s) \nu(d v)>v\left(O s^{-1}\right)-\frac{1}{2^{n}} .
$$

The set $B_{s}=\left\{z \mid \int f_{s}(v z) \nu(d v)>k / 2^{n}-1 / 2^{n}\right\}$ is an open set containing $s$. Hence the sets $B_{s}, s \in C_{k, n}$, are an open covering of $C_{k, n}$. There is a finite subcovering $B_{s_{1}}, \cdots, B_{s, j}$ of $C_{k, n}$. Let $f_{k, n}(s)=\max _{i=1, \ldots, j} f_{s_{i}}(s)$. Clearly

$$
\int f_{k, n}(v z) \nu(d v)>\frac{k-1}{2^{n}}
$$

for all $z \in C_{k, n}$. Further $f_{k, n} \in \eta, f_{k, n}=0$ on $\bar{D}$. In this way we obtain such a function $f_{k, n}$ for $C_{k, n}, k=0,1, \cdots, 2^{n}$. Let $f(s)$ $=\max _{k=0,1}, \ldots, 2^{n} f_{k, n}(s)$. Then

$$
\begin{aligned}
\int\left\{\int f(v z) \nu(d v)\right\} \mu(d z) & >\sum_{k=0}^{2^{n}} \frac{k-1}{2^{n}} \mu\left(C_{k, n}\right) \\
& >\int \nu\left(O s^{-1}\right) \mu(d s)-\frac{2}{2^{n}}-\frac{\epsilon}{2}>\int \nu\left(O s^{-1}\right) \mu(d s)-\epsilon
\end{aligned}
$$

where $f \in \eta, f=0$ on $\bar{O}$. Since this holds for any $\epsilon>0$, we have the desired conclusion for open sets.

Let $F_{n}\left(F_{n} \subset A\right), O_{n}\left(A \subset O_{n}\right)$ be nondecreasing and nonincreasing sequences of closed and open sets such that $\nu * \mu\left(F_{n}\right), \nu * \mu\left(O_{n}\right)$ 
$\rightarrow \nu * \mu(A)$ as $n \rightarrow \infty$ for a fixed $A \in B(S)$. The existence of such sequences follows from the regularity of $\nu * \mu$. But then

$$
\begin{aligned}
\nu * \mu(A) & =\lim _{\star} \nu * \mu\left(O_{n}\right)=\lim _{n} \int \nu\left(O_{n} s^{-1}\right) \mu(d s) \\
& \geqq \int_{n} \nu\left(A s^{-1}\right) \mu(d s) \geqq \lim _{*} \int\left(F_{n} s^{-1}\right) \mu(d s) \\
& =\lim _{n} \nu * \mu\left(F_{n}\right)=\nu * \mu(A) .
\end{aligned}
$$

Thus Lemma 3 holds for general $A \in \mathbb{B}(S)$.

Suppose $\mu$ is an idempotent measure on $S$. Then

$$
\begin{aligned}
\mu\left(A s^{-1}\right) & =\int \mu\left(A s^{-1} s^{\prime-1}\right) \mu\left(d s^{\prime}\right)=\int \mu\left(A\left(s^{\prime} s\right)^{-1}\right) \mu\left(d s^{\prime}\right) \\
& =\int \mu\left(A s^{\prime-1}\right) \mu\left(d s^{\prime} s^{-1}\right)
\end{aligned}
$$

By Theorem 14 of [3] we already know that an idempotent probability measure must have a completely simple semigroup as its support. From this point on let us take $S$ a compact completely simple semigroup with representation $T \times X \times Y$ and corresponding function $\phi$.

Suppose $\mu$ is an idempotent measure on $S$ with support $S$. Let

$$
S_{x}=\{s \mid x(s)=x\},
$$

that is, $S_{x}$ is the subset of points in $S$ whose $x$ coordinate $x(s)$ in representation ( ) of the semigroup is the fixed point $x$ in $X$. Then $P(s, A)=\mu\left(A s^{-1}\right)$ is an idempotent Markov transition measure (see [4]) for $A \in \mathbb{B}\left(S_{x}\right), s \in S_{x}$, that is

$$
P(s, A)=\int_{S_{x}} P\left(s, d s^{\prime}\right) P\left(s^{\prime}, A\right) .
$$

$\mathrm{B}\left(S_{x}\right)$ is the Borel field on $S_{x}$ induced by $B(S)$. We shall call $B \in \mathbb{B}\left(S_{x}\right)$ an invariant set if

$$
P(s, B)=1
$$

for all $s \in B$. We say that $S_{x}$ is irreducible if one cannot find two disjoint nonvacuous sets $A, B \in \mathbb{B}\left(S_{x}\right)$ such that

$$
\begin{array}{ll}
P(s, A) \equiv 1 & \text { for all } s \in A, \\
P(s, B) \equiv 1 & \text { for all } s \in B .
\end{array}
$$


LEMMA 4. Let $\mu$ be a regular idempotent probability measure with support the compact completely simple semigroup $S$. Then $S_{x}$ (for every $x \in X)$ is irreducible with respect to $P(s, A)=\mu\left(A s^{-1}\right), A \in B\left(S_{x}\right)$, $s \in S_{x}$.

Suppose $S_{x}$ is not irreducible. Then there are two disjoint nonvacuous invariant sets $A, B \in \mathbb{B}\left(S_{x}\right)$. Both these invariant sets must be dense in $S_{x}$. For consider any invariant set $A$. Let $s$ be any point of $S_{x}$. Consider any open neighborhood $N$, of $s$ and take $a$ any point of $A . N_{8} a^{-1}$ is open by the continuity of the multiplicative operation. But then $P\left(a, N_{s}\right)=\mu\left(N_{s} a^{-1}\right)>0$ and hence $N_{s}$ contains an element of $A$. Thus $A$ is dense in $S_{x}$.

Let $a$ be an element of $A$. By the regularity of $\mu\left(\cdot a^{-1}\right)$ on $S_{x}$ there is a closed set $C \subset A$ such that $\mu\left(C a^{-1}\right)>1-\epsilon>0$. But $S_{x}-C$ is open in $S_{x}$ and contains $B . B$ is dense in $S_{x}$ so that $S_{x}-C$ is all of $S_{x}$. However, this contradicts $\mu\left(C a^{-1}\right)>0$.

LeммA 5. Let $\mu$ be an idempotent probability measure with support the compact completely simple semigroup $S$. Then $P(s, A)=\mu\left(A s^{-1}\right)$ with $s \in S_{x}, A \in \mathbb{B}\left(S_{x}\right)$ is independent of $s$.

Consider $P(s, A)=\mu\left(A s^{-1}\right)$ with $s \in S_{x}, A \in B\left(S_{x}\right)$. Let

$$
f(s)=\int_{S_{x}} P\left(s, d s^{\prime}\right) f\left(s^{\prime}\right)
$$

for $f$ a bounded function on $S_{x}$ measurable with respect to $P(s, \cdot)$ for every $s$. Since $P(s, \cdot)$ is an idempotent transition probability function (see (7))

$$
\int_{S_{x}} P\left(s, d s^{\prime}\right)\left[\bar{f}\left(s^{\prime}\right)-f\left(s^{\prime}\right)\right] \equiv 0 .
$$

We shall call $f(s)$ an almost invariant function if the set $E_{f}$ $=\left\{s^{\prime} \mid f\left(s^{\prime}\right) \neq \bar{f}\left(s^{\prime}\right)\right\}$ is of zero $P(s, \cdot)$ measure for every $s$. Consider a bounded function $f$ such that

$$
\bar{f}(s) \geqq f(s)
$$

except possibly for a set $G_{f}$ of zero $P(s, \cdot)$ measure for every $s$. Such a function is an almost invariant function since by (11) the set on which $f(s) \neq \bar{f}(s)$ is of zero $P(s, \cdot)$ measure for all $s$.

If $f, g$ are almost invariant, then $\max (f, g)$ is almost invariant. The set of almost invariant functions is a linear space and is closed under bounded pointwise convergence. This implies that if $f$ is an almost invariant function then for any fixed $\alpha$ the characteristic function $c_{A_{\alpha}}(s)$ of the set 


$$
A_{\alpha}=\{s \mid f(s)>\alpha\}
$$

is almost invariant. For consider $g(s)=\max (f(s)-\alpha, 0)$ which is almost invariant. Let $h_{n}(s)=\min (n g(s)-1,0)+1$. But $c_{A_{\alpha}}(s)$ $=\lim _{n} h_{n}(s)$, that is, it is the limit of almost invariant functions and hence almost invariant. Thus, except for a set $E$ which is of $P(s, \cdot)$ measure zero for every $s, c_{A_{\alpha}}(s)=\bar{c}_{A_{\alpha}}(s)$. If $s \in A_{\alpha}-E$ then

$$
P\left(s, A_{\alpha}-E\right)=P\left(s, A_{\alpha}\right)=1 .
$$

Similarly if $s \in \bar{A}_{\alpha}-E$ ( $\bar{A}$ is the complement of $A$ ) then

$$
P\left(s, \bar{A}_{\alpha}-E\right)=P\left(s, \bar{A}_{\alpha}\right)=1 .
$$

Thus, if they are nonvacuous, $A_{\alpha}-E$ and $\bar{A}_{\alpha}-E$ are invariant sets.

Now consider setting $f(s)=P(s, A)$ for any fixed $A \in \mathbb{B}\left(S_{x}\right)$. Clearly $P(s, A)$ is almost invariant. The argument given above implies that there is an $\alpha$ such that

$$
P(s, A) \equiv \alpha
$$

for all $s$ except those in a set $E_{A}$ of $P(s, \cdot)$ measure zero for every $s$. But then

$$
P(s, A)=\int_{S_{x}} P\left(s, d s^{\prime}\right) P\left(s^{\prime}, A\right)=\int_{E_{A}} P\left(s, d s^{\prime}\right) P\left(s^{\prime}, A\right) \equiv \alpha
$$

for all $s$, whether outside $E_{A}$ or in $E_{A}$. The proof of Lemma 5 is complete.

We are now ready to finish the proof of the theorem. Let us look at

$$
\mu\left((U \times V \times W) s^{-1}\right)
$$

where $U \times V \times W$ is a product set with $U \in \mathbb{B}(T), V \in \mathbb{B}(X), W \in \mathbb{B}(Y)$. Notice that if (18) is positive we must have $x(s) \in V$. Now

(19) $(U \times V \times W) s^{-1}=\left\{s^{\prime} \mid t\left(s^{\prime}\right) \in U t\left(s^{-1} \phi\left(x\left(s^{\prime}\right), y(s)\right)^{-1}, y\left(s^{\prime}\right) \in W\right\}\right.$.

Lemma 5 implies that (18) is independent of $s$ for which $x(s) \in V$ and therefore by (19)

$$
\mu\left((U \times V \times W) s^{-1}\right)=\mu\left((U t \times V \times W) s^{-1}\right)
$$

for all $t \in T$ if $x(s) \in V$. Expression (18) is zero if $x(s) \notin V$. But this implies that

$$
\mu\left((U \times V \times W) s^{-1}\right)=\chi(U) \mu\left((T \times V \times W) s^{-1}\right)
$$

where $\chi$ is the normed Haar measure of $T$ (the support of (18) with $V, W$ fixed and $\mu(T \times V \times W)>0$ is all of $T)$. However 


$$
\mu\left((T \times V \times W) s^{-1}\right)=\beta(W)
$$

if $x(s) \in V$ and zero otherwise. Now

$$
\begin{aligned}
\mu((U \times V \times W)) & =\int \mu\left((U \times V \times W) s^{-1}\right) \mu(d s) \\
& =\int_{x(s) \in V} \chi(U) \beta(W) \mu(d s)=\chi(U) \alpha(V) \beta(W) .
\end{aligned}
$$

The proof of the theorem is complete.

\section{REFERENCES}

1. N. Dunford and J. Schwartz, Linear operators, Vol. I, Interscience, New York, 1958.

2. P. R. Halmos, Measure theory, Van Nostrand, New York, 1951.

3. B. M. Kloss, Probability distributions on bicompact topological groups, Theor. Probability Appl. 4 (1959), 237-270.

4. M. Loeve, Probability theory, Van Nostrand, New York, 1961.

5. M. Rosenblatt, Limits of convolution sequences of measures on a compact topological semigroup, J. Math Mech. 9 (1960), 293-306.

6. A. D. Wallace, The Rees-Suschkewitsch structure theorem for compact simple semigroups, Proc. Nat. Acad. Sci. U.S.A. 42 (1956), 430-432.

SYRACUSE UNIVERSITY AND

BROWN UNIVERSITY 